Hauke, Alexander; Krämer, Juliane; Laqua, Daniel; Ley, Sebastian ; Husar, Peter:

High performance triple LED driver with digitally controlled analog dimming for reflection pulse oximetry

Zuerst erschienen in: $\quad$ Biomedical Engineering = Biomedizinische Technik. - Berlin [u.a.] : de Gruyter. - 57 (2012), Suppl. 1, Track-E, p. 580-582.

Erstveröffentlichung: $\quad$ 2012-08-30

ISSN (online): $\quad$ 1862-278X

ISSN (print): $\quad$ 0013-5585

DOI: $\quad 10.1515 / \mathrm{bmt}-2012-4128$

[Zuletzt gesehen: 2019-08-12]

„Im Rahmen der hochschulweiten Open-Access-Strategie für die Zweitveröffentlichung identifiziert durch die Universitätsbibliothek IImenau. “

"Within the academic Open Access Strategy identified for deposition by Ilmenau University Library."

„Dieser Beitrag ist mit Zustimmung des Rechteinhabers aufgrund einer (DFGgeförderten) Allianz- bzw. Nationallizenz frei zugänglich."

"This publication is with permission of the rights owner freely accessible due to an Alliance licence and a national licence (funded by the DFG, German

DFG

Research Foundation) respectively." 


\title{
High Performance Triple LED Driver with Digitally Controlled Analog Dimming for Reflection Pulse Oximetry
}

\author{
A. Hauke ${ }^{1}$, J. Krämer ${ }^{2}$, D. Laqua ${ }^{2}$, S. Ley ${ }^{2}$ and P. Husar ${ }^{2}$ \\ ${ }^{1}$ Institute of Biomedical Engineering and Informatics, Ilmenau University of Technology, 98693 Ilmenau, Germany, \\ alexander.hauke@tu-ilmenau.de \\ ${ }^{2}$ Institute of Biomedical Engineering and Informatics, Ilmenau University of Technology, 98693 Ilmenau, Germany
}

\begin{abstract}
This contribution describes the development of a prototype that improves standard pulse oximetry to deep tissue penetration. It shows the setup of a control unit operating a high power triple LED driver with a high throughput of electrical power and governing a time table used for proper LED toggeling. To match the depth of light penetration for each used wavelength the possibility of digitally controlled analog dimming has been applied. In order to penetrate human tissue it was important to choose the right wavelengths for the LEDs. For proper data acquisition an active sensor module was designed, using a photodiode and a high resolution ADC. Results show how the prototype's functionality could be established and first measurements were taken successfully.
\end{abstract}

\section{Introduction}

As reflection pulse oximetry is a non-invasive method to determine the oxygenation of blood in superficial tissue [1], this contribution is to describe the development of a prototype that improves standard reflection pulse oximetry to deep tissue light penetration. It is described how a control unit is set up to operate a high power triple LED driver for sufficiently supplying high performance LEDs. To ensure a sufficient depth of penetration choosing the right wavelengths was as crucial, as an intense emission of light.

\section{Methods}

\subsection{Concept}

The design of the prototype shown in Figure 1 is aligned to traditional configurations of pulse oximeters, but using a triple LED driver for employing a third wavelength instead of two, as used in common pulse oximeters [2].

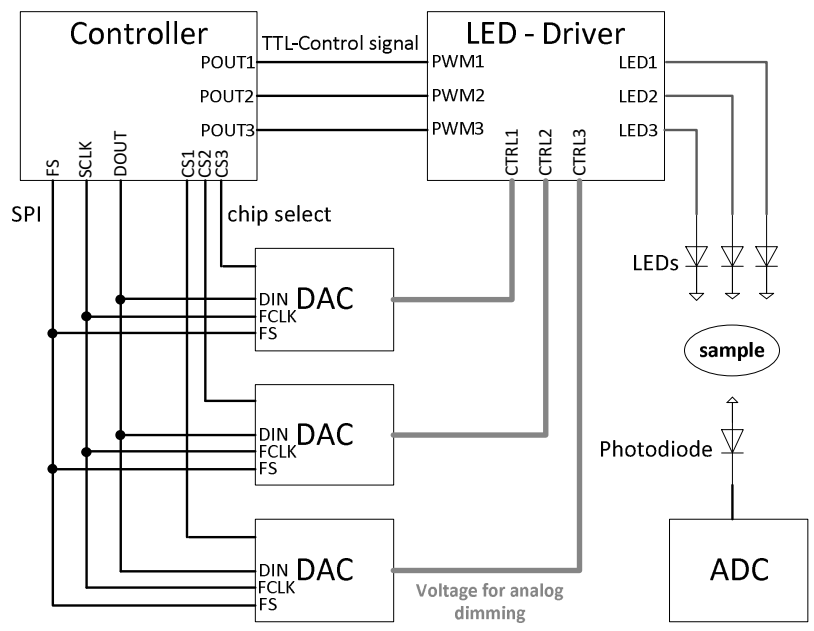

Figure 1: Concept of design of the prototype. The control unit governs the behavior of the LED driving unit and several DACs and therefore holds control over LED timing and analog dimming. Data acquisition is carried out by an ADC.

\subsection{Controller}

\subsubsection{Analog Dimming}

In this concept the control unit provides the possibility of analog dimming by forcing constant output voltages of three DACs, which are programmed via SPI. By using chip select each DAC may be programmed differently. These analog voltages are used to determine the output currents of the LED driver for each LED string.

\subsubsection{Timing}

Furthermore the controller governs a strict timing table shown in Figure 2.

This timing is directly provided to the LED driver's PWM input pins by a TTL-Control signal. As shown above each LED is switched on while the others stay turned off. After a short dead time, which is to avoid an overlap of runtimes of LEDs of different wavelengths, the next LED is turned on. The fourth period after switching off the third LED is used to eliminate the influences of diffused light during the data processing later on.

The timing table can be digitally adjusted to fit different purposes or new conditions of measurement.

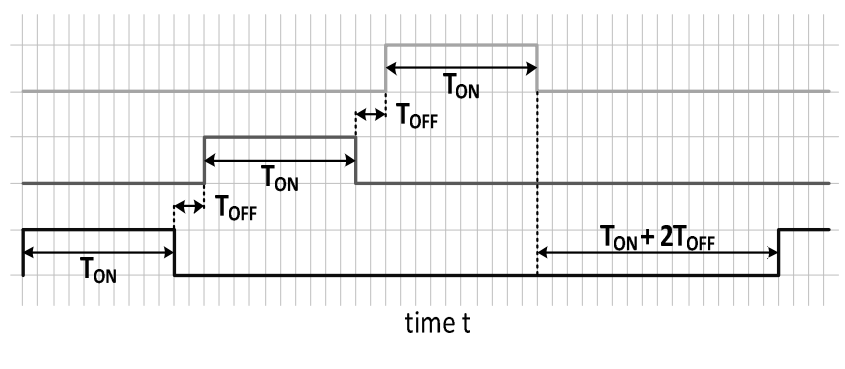

$$
\text { - LED } 1 \text { - LED } 2 \text { - LED } 3
$$

Figure 2: Timing governed by the control unit to ensure precise 
switching times for the LEDs. $\mathrm{T}_{\mathrm{ON}}$ determines the runtimes of the LEDs, $\mathrm{T}_{\mathrm{OFF}}$ determines their off periods before switching on the next LED.

\subsection{LED - Driver}

In order to achieve an intense emission of light for deep tissue penetration, high performance LEDs had to be supplied sufficiently. Therefore a triple LED step down driver with a high throughput of electrical power was chosen. Its output current follows the analog voltages provided by the DACs linearly. So by digitally adjusting the DACs analog output voltages the LEDs brightness can be directly influenced by increasing or decreasing the LED driver's output currents. As each output current can be adjusted independently, it is possible to set each LED string to a different brightness.

Though the timing signals of the controller are applied to the LED drivers PWM input pins the output is not regulated by an actual PWM signal. As the control signal is a digital signal, the LED driver switches its output on or off, according to the timing table. When turned off the driver operates as a current sink.

\subsection{LEDs}

To achieve a sufficient depth of light penetration it is recommended to make use of wavelengths in the near infrared and infrared spectrum. According to this, wavelengths of $720 \mathrm{~nm}, 810 \mathrm{~nm}$ and $905 \mathrm{~nm}$ were chosen for the LEDs.

Figure 3 shows the isobestic point, which marks the wavelength in the absorption spectrums of fetal and adult blood, at which the absorption of light is exactly the same for both types of blood. Measurements at this wavelength can be taken as a point of reference in further data processing. To avoid an overlapping of the LEDs spectrums by temperature related drift, the remaining wavelengths were chosen with a distance of about $\pm 100 \mathrm{~nm}$ to the isobestic point.

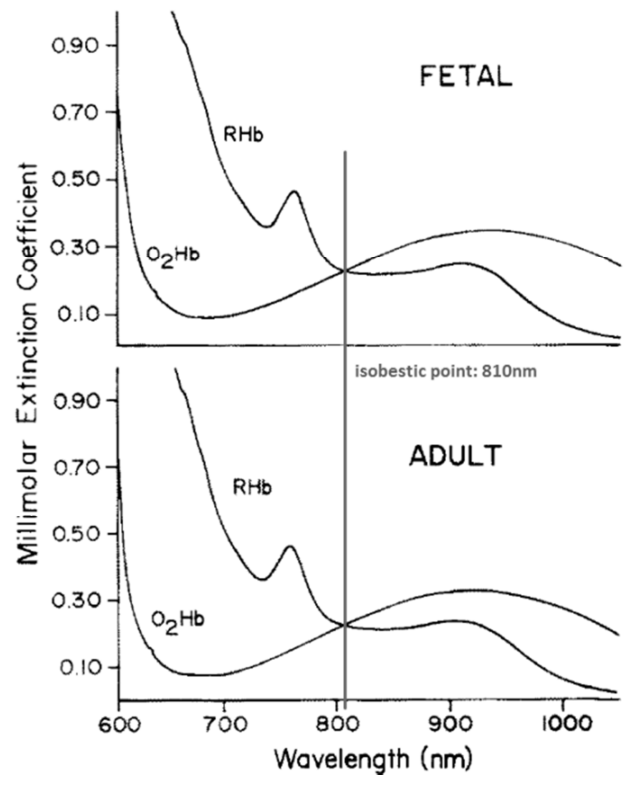

Figure 3: Absorption spectrums of fetal and adult blood, comparing oxygenated and deoxygenated hemoglobin [3].

\subsection{Sensor}

The active sensor module consists of a photodiode, matched to the used wavelengths and a high resolution ADC with sample rates up to $50 \mathrm{ksps}$.

\section{Results}

The prototype's functionality was established and successfully confirmed by several measurements.

\subsection{Analog Dimming}

Figure 4 proves the LEDs' brightness could be adjusted digitally. It shows the influence of a variation of the LED drivers output currents on the output voltage of the photodiode. The graph of the $810 \mathrm{~nm}$ measurements reaches a plateau at about $4.5 \mathrm{~V}$ because the photodiode's maximum output voltage was reached.

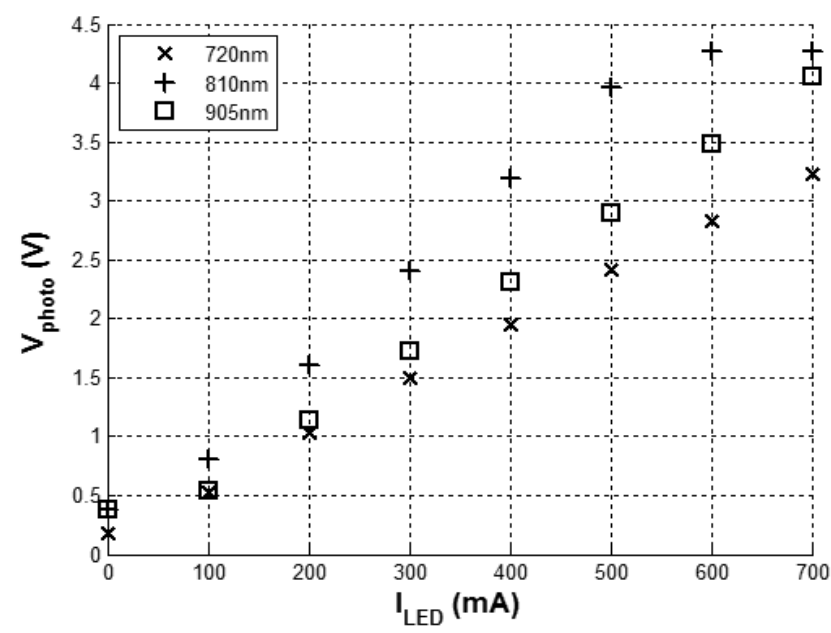

Figure 4: Output voltage of the photodiode compared to the LEDs' input current measured at a distance of $20 \mathrm{~cm}$.

\subsection{Timing}

Figure 5 gives prove about the correct pulsation of the LEDs, according to the time table given in Figure 2.

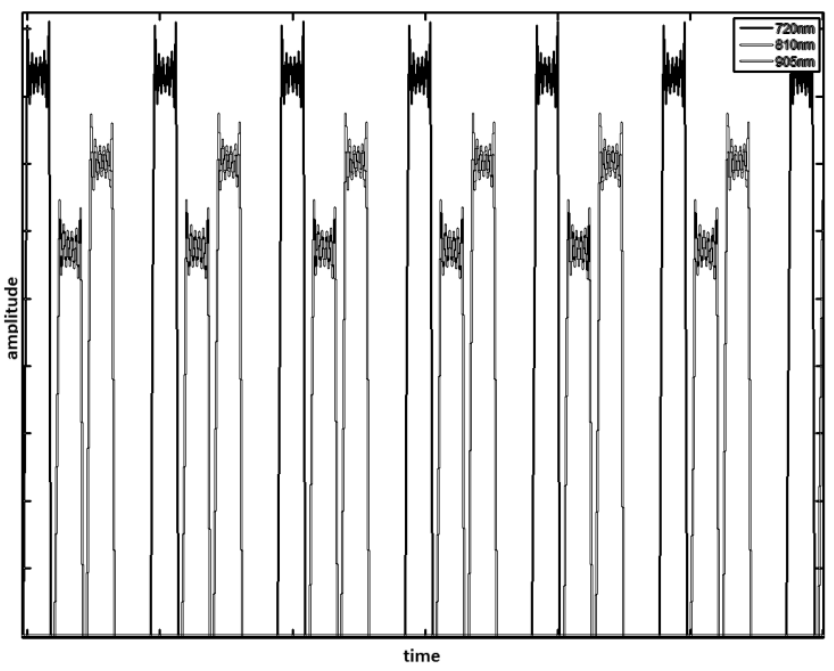

Figure 5: Pulsation of the LEDs' voltage measured on a digital oscilloscope. 


\subsection{Pulse curve}

First measurements were taken of the abdominal region of several subjects. To avoid detecting pulse curves from superficial tissue the LEDs were placed on the left abdominal region and the sensor on the right. Like this the effect of light travelling through superficial tissue should be minimized, and the effect of light reflected from deeper tissue maximized [4]. Figure 6 shows a $10 \mathrm{~s}$ sample of pulse curves, detected for each wavelength with normalized amplitudes. The graphs prove the prototype working, as pulse curves could clearly be detected. The deviation of amplitudes can be explained by different intensities of light and the different wavelengths abilities to penetrate deeper tissue. In further measurements this effect could be avoided by taking the prototype's ability for analog dimming into account. As data processing was carried out offline with MATLAB it wasn't possible to adjust the LEDs' brightness during the first measurements.

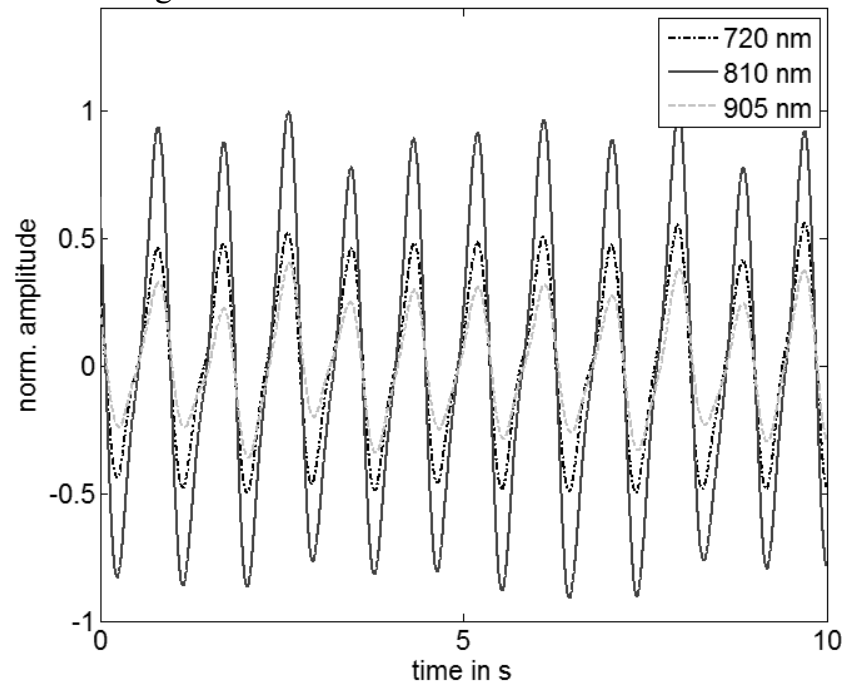

Figure 6: Pulse curve detected from the abdominal region of a subject.

\section{Conclusion}

As this prototype made it possible to detect pulse curves from deeper abdominal regions, it offers use in several applications, as conventional and non-invasive fetal reflection pulse oximetry. To improve measurement a dynamic light intensity governing could be applied to enable compensation of the amplitudes deviation online.

\section{$5 \quad$ References}

[1] C. Marx, "Geburtshilfe: Fetale Pulsoxymetrie," Deutsches Ärzteblatt, vol. 63, no. 61, pp. 140144, 2003.

[2] J. G. Webster, Ed., Design of Pulse Oximeters. Institute of Physics Publishing, 1997.

[3] M. Andrew P. Harris, MD, Michael J. Sendak, P. Robert T. Donham, MD, and P. Michael Thomas, $\mathrm{PhD}$, and Donald Duncan, "ABSORPTION CHARACTERISTICS OF HUMAN FETAL HEMOGLOBIN AT WAVELENGTHS USED IN PULSE OXIMETRY,” pp. 175-177, 1988.

[4] A. Zourabian, A. Siegel, B. Chance, N. Ramanujan, M. Rode, and D. a Boas, "Trans-abdominal monitoring of fetal arterial blood oxygenation using pulse oximetry.," Journal of biomedical optics, vol. 5, no. 4, pp. 391-405, Oct. 2000. 